\title{
Clinical characteristics and outcomes of ocular allergy in Thai children
}

\author{
Rasamee Jongvanitpak, ${ }^{1}$ Pakit Vichyanond, ${ }^{1}$ Orathai Jirapongsananuruk, ${ }^{1}$ Nualanong Visitsunthorn, ${ }^{1}$ Punchama Pacharn ${ }^{1}$
}

\section{Abstract}

Background: Allergic conjunctivitis (AC) is the most common ocular condition in allergic children. In tropical countries, the study about the clinical features and outcome of treatment is very limited.

Objective: To review clinical characteristics and outcomes of treatment in children with ocular allergy.

Methods: Children with history of AC were classified to seasonal allergic conjunctivitis (SAC), perennial allergic conjunctivitis (PAC), vernal keratoconjunctivitis (VKC) and atopic keratoconjunctivitis (AKC). The clinical history and outcome of treatment were recorded.

Results: One hundred and sixty-four children were recruited. PAC was the most common type (61.6\%), followed by SAC (21.3\%), VKC (12.2\%), and AKC (4.9\%). Male preponderance was found in all groups. Mean age of onset was $6.8 \pm 2.8$ years. Allergic rhinitis was the most common co-morbidity (97.6\%). The common sensitized allergen is house-dust mites $(86.1 \%)$. Standard treatments in all groups were natural tear and topical olopatadine. Add-on medications were usually needed in severe types of AC (VKC, AKC). History of topical corticosteroid use was 68.8\% and $12.5 \%$ in VKC and AKC, respectively. All of them can discontinue topical corticosteroid when topical tacrolimus was applied. The overall remission was found 35\% in VKC group and 63\% in AKC group. The median duration of treatment was 20.5 months in VKC group and 11 months in AKC group.

Conclusion: most Thai children with AC sensitized to house-dust mites. In severe forms of AC, most patients needed add-on medication. The use of topical calcineurin inhibitor as an add-on therapy can decrease the use of topical corticosteroid.

Key words: conjunctivitis, allergic conjunctivitis, keratoconjunctivitis, treatment outcome, tacrolimus

\section{Citation:}

Jongvanitpak, R., Vichyanond, P., Jirapongsananuruk, O., Visitsunthorn, N., Pacharn, P. (2022). Clinical characteristics and outcomes of ocular allergy in Thai children.

Asian Pac J Allergy Immunol, 40(4), 407-413.

https://doi.org/10.12932/ap-160519-0564

\section{Affiliation:}

Division of Allergy and Immunology, Department of Pediatrics, Faculty of Medicine Siriraj Hospital, Mahidol University, Bangkok, Thailand

\section{Corresponding author:}

Punchama Pacharn

Division of Allergy and Immunology, Department of Pediatrics

Faculty of Medicine Siriraj Hospital, Mahidol University

2 Prannok Road, Bangkoknoi, Bangkok 10700, Thailand

E-mail: punchama@gmail.com

\section{Introduction}

Allergic conjunctivitis is one of the most common ocular conditions in general pediatric practices. The prevalence of allergic conjunctivitis varied depending on the criteria of diagnosis and study population. The data from NHANESIII found that among 20,010 samples, $6.4 \%$ reported the ocular symptom alone, and $29.7 \%$ reported both ocular and nasal symptoms. ${ }^{1}$ In children, the largest epidemiologic study on prevalence of allergic diseases was performed by the International Study of Asthma and Allergies in Childhood (ISAAC). Unfortunately, the data from ISAAC reported conjunctivitis in combination with rhinitis. From the reported of ISAAC phase three, the prevalence of rhinoconjunctivitis in Asia-Pacific region was 3.6-17.7\% in 6-7 year-old children, and $4.8-21 \%$ in $13-14$ year-old children. ${ }^{2}$ The ISAAC group was continued as The Global Asthma Network (GAN) from 2012. The GAN phase I study in Thailand found that among 6,291 children, $16.6 \%$ reported current eye symptoms. ${ }^{3}$ 
Allergic conjunctivitis (AC) in children can be categorized into four types, which are seasonal allergic conjunctivitis (SAC), perennial allergic conjunctivitis (PAC), vernal keratoconjunctivitis (VKC) and atopic keratoconjunctivitis (AKC). ${ }^{4}$ The mild form of AC (SAC and PAC) usually presented with eye itching, redness and tearing. ${ }^{5,6}$ Whereas the severe form (VKC and AKC), patients usually have more severe of the symptoms including photophobia, characteristic ropy discharge, and can develop complication such as corneal ulcer or sight-threatening condition. ${ }^{7}$ On physical examination, giant papillae at conjunctiva and Horner Trantas dot can be found in both severe form of AC. The severity of symptoms depend on many factors such as type and degree of allergen sensitization, seasonality, weather, infection. ${ }^{8}$ Therefore, patients who live in different climate may have different characteristics of the diseases and prognosis.

According to Almaliotis study, ${ }^{9}$ skin prick tests were shown positive around two-thirds of the ocular allergic patients (76.8\%) and the majority of aeroallergen sensitizations were grass pollen (58.5\%), followed by dust mites (43.7\%), cat dander (23.6\%) and dog dander (12.3\%). In Thailand, the study in patients with $\mathrm{AC}$ found that the most common allergen was house-dust mites (70\%), followed by cockroaches (44\%) and grass (43\%). ${ }^{10}$ So the type of aeroallergen is different among regions.

The outcome of the severe form of AC is also crucial because it can affect the eye sight. The mechanisms of the severe form are both $\operatorname{IgE}$ and non IgE-mediated hypersensitivity. T helper 2 cells and their cytokines, corneal fibroblasts and epithelium along with various growth factors play an important role in the pathogenesis of VKC. ${ }^{4,11}$ As a result, the standard treatment is usually not effective in the severe form. Add-on medication such as topical calcineurin inhibitor (tacrolimus), cyclosporin or corticosteroid were helpful to control eye symptoms in this group of patients. ${ }^{7,812-14}$ Because the use of topical corticosteroid may cause complication such as glaucoma or cataract, so it should be avoided for these patients.

Thailand located in tropical area that might affect the symptoms, severity and aeroallergen sensitization in ocular allergy. The previous study in Thailand ${ }^{10}$ recruited mostly adult population (mean age $24.5 \pm 16.3$ years) and they did not evaluate the outcome of the treatment. Therefore, this study aims to analyze aeroallergen sensitization in Thai children with ocular allergy. Also, we will assess the clinical manifestation and the efficacy of treatment, focused on severe forms of allergic conjunctivitis (both VKC and AKC).

\section{Materials and Methods Subjects}

The retrospective medical records review of the children with a history of allergic conjunctivitis, aged 4-18 years, who visited at the pediatric allergy clinic of Siriraj Hospital between Jan 2014 and Feb 2015 were performed. This study enrolled the patients after the Siriraj Institutional Review Board (SIRB) was approved for the study protocol (COA no. si056/2014). The demographic data, family history, results of skin prick tests, type of ocular allergy, and outcome of the treatment were recorded. Patients who had infectious conjunctivitis were excluded. SAC is defined as acute, bilateral, self-limiting allergic conjunctivitis occurs seasonally (spring, fall). ${ }^{2,14}$ However, Thailand is a tropical country, so the climate is divided into 3 seasons (summer, rainy and cool). PAC is defined as chronic, bilateral, self-limiting allergic conjunctivitis occur year-round. ${ }^{2,14} \mathrm{VKC}$ is defined as bilateral chronic allergic conjunctivitis and develops a papillary response, principally manifesting in the limbus or upper tarsus. ${ }^{14}$ AKC is defined as bilateral, chronic allergic conjunctivitis and periocular dermatitis. ${ }^{14}$ This study was registered to the Thai Clinical Trials Registry (TCTR20190528001).

\section{Skin-test procedure}

Skin prick tests (SPT) were performed with commercial aeroallergen extracts (ALK, Abello, Sweden) including house dust mites (D. pteronyssinus, D. farinae), cockroaches (American spp., German spp.), animal dander (dog, cat), pollens (Bermuda, Johnson, Acacia, Careless weed) and molds (Penicillium spp., Aspergillus spp., Furasium spp., Currvularia spp., Alternaria spp., Cladosporium spp.). Histamine phosphate $(10 \mathrm{mg} / \mathrm{mL})$ and glycerinated saline were used as positive and negative control, respectively. The mean wheal diameter (MWD) was calculated from the sum of the largest measurement across the wheal and divided by two. SPT was considered positive if the MWD was $\geq 3 \mathrm{~mm}$ compared to negative control.

\section{Outcome of treatment}

In severe forms of allergic conjunctivitis (VKC, AKC), remission with standard treatment is defined as patients who self-limiting symptoms without using topical calcineurin inhibitor, complete remission is defined as patients who can discontinue topical tacrolimus eye ointment more than 1 year without any symptoms. Partial remission is defined as relapsing condition during cessation period of topical tacrolimus eye ointment. ${ }^{15}$ Topical $0.1 \%$ FK-506 eye ointment was prepared as previously described. ${ }^{8}$ Giant papillary injection is defined as papillary injection size $>1 \mathrm{~mm} .{ }^{16}$

\section{Statistical analysis}

Data were analyzed using the IBM SPSS 18.0 program ((IBM Co., Armonk, NY, USA.SPSS Inc). An unpaired t-test or the Mann-Whitney $U$ test for quantitative data and chi-square test for qualitative data were used to compare the data between 2 groups. Statistical significance was denoted by a $p$ value of less than 0.05 .

\section{Results}

One hundred and sixty-four patients, aged 4-18 years, were reviewed. The demographic data of these patients are shown in Table 1. PAC was the most common types of AC (61.6\%), followed by SAC (21.3\%), VKC (12.2\%), and AKC (4.9\%). Mean age of onset of overall AC was $6.8 \pm 2.8$ years whereas the mean age of diagnosis was $7.8 \pm 2.8$ years. VKC seems to have earlier onset $(5.8 \pm 2.4$ years $)$ than the others. 
Table 1. Demographic and clinical characteristics of allergic conjunctivitis patients $(\mathrm{N}=164)$

\begin{tabular}{|c|c|c|c|c|c|}
\hline Characteristics & $\begin{array}{c}\text { SAC } \\
(n=35)\end{array}$ & $\begin{array}{c}\text { PAC } \\
(n=101)\end{array}$ & $\begin{array}{c}\text { VKC } \\
(n=20)\end{array}$ & $\begin{array}{c}\text { AKC } \\
(\mathbf{n}=\mathbf{8})\end{array}$ & $p$ \\
\hline Age of onset (years) & $7.2 \pm 2.9$ & $6.8 \pm 2.8$ & $5.8 \pm 2.4$ & $7.4 \pm 2.6$ & 0.33 \\
\hline Age of diagnosis (years) & $7.6 \pm 3.1$ & $7.8 \pm 2.8$ & $7.9 \pm 2.7$ & $8.8 \pm 3.0$ & 0.75 \\
\hline Gender: Male & $23(65.7 \%)$ & $63(62.4 \%)$ & $17(85.0 \%)$ & $6(75.0 \%)$ & 0.26 \\
\hline Family history of atopy & $20(57.1 \%)$ & $67(66.3 \%)$ & $6(30.0 \%)$ & $7(87.5 \%)$ & 0.007 \\
\hline \multicolumn{6}{|l|}{ Comorbidity } \\
\hline Allergic rhinitis & $35(100 \%)$ & $100(99.0 \%)$ & $17(85.0 \%)$ & $8(100 \%)$ & 0.02 \\
\hline Asthma & $20(57.1 \%)$ & $41(40.6 \%)$ & $5(25.0 \%)$ & $3(37.5 \%)$ & 0.12 \\
\hline Atopic dermatitis & $2(5.7 \%)$ & $20(19.8 \%)$ & $3(15.0 \%)$ & $8(100 \%)$ & $<0.001$ \\
\hline Positive skin prick test & $27(77.1 \%)$ & $93(92.1 \%)$ & $15(75.0 \%)$ & $8(100 \%)$ & 0.05 \\
\hline
\end{tabular}

Data presented as mean \pm standard deviation or number and percentage

$p$-value $<0.05$ indicates statistical significance

Abbreviations: SAC, seasonal allergic conjunctivitis; PAC, perennial allergic conjunctivitis; VKC, vernal keratoconjunctivitis; AKC, atopic keratoconjunctivitis

Male preponderance was found in all groups. Most of them have family history of atopy except VKC (30\%). Allergic rhinitis was the most common co-morbidity (160; 97.6\%) followed by asthma $(69 ; 42.1 \%)$, and atopic dermatitis (33; $20.1 \%)$.

In seasonal allergic conjunctivitis, most of them have typically exacerbation during rainy season (57.1\%).

\section{Clinical manifestations}

The most common presentation of patients with ocular allergy is eye itching, followed by allergic shiner, and hyperemia. (Table 2). Photophobia is found only in severe form (VKC, AKC) of ocular allergy. Those with severe form usually present with eye itching, hyperemia, and photophobia. Among 20 patients with VKC, 18 of them

Table 2. Clinical manifestations compared among the different types of allergic conjunctivitis

\begin{tabular}{|c|c|c|c|c|c|}
\hline Manifestations & $\begin{array}{c}\text { SAC } \\
(n=35)\end{array}$ & $\begin{array}{c}\text { PAC } \\
(n=101)\end{array}$ & $\begin{array}{c}\text { VKC } \\
(n=20)\end{array}$ & $\begin{array}{c}\text { AKC } \\
(n=8)\end{array}$ & $p$ \\
\hline Itchy eye, $\mathrm{n}(\%)$ & $35(100 \%)$ & $101(100 \%)$ & $20(100 \%)$ & $8(100 \%)$ & - \\
\hline Red eye, n (\%) & $29(82.9 \%)$ & $87(86.1 \%)$ & $18(90.0 \%)$ & $8(100 \%)$ & 0.61 \\
\hline Lacrimation, n (\%) & $0(0.0 \%)$ & $12(11.9 \%)$ & $14(70.0 \%)$ & $1(12.5 \%)$ & $<0.001$ \\
\hline Photophobia, n (\%) & $0(0.0 \%)$ & $0(0.0 \%)$ & $15(75.0 \%)$ & $4(50.0 \%)$ & $<0.001$ \\
\hline Allergic shiner, n (\%) & $33(94.3 \%)$ & $97(96.0 \%)$ & $13(65.0 \%)$ & $8(100 \%)$ & $<0.001$ \\
\hline \multicolumn{6}{|l|}{ Papillae, n (\%) } \\
\hline Fine & $27(77.1 \%)$ & $100(99.0 \%)$ & $13(65.0 \%)$ & $7(87.5 \%)$ & $<0.001$ \\
\hline Giant & $0(0.0 \%)$ & $0(0.0 \%)$ & $11(55.0 \%)$ & $0(0.0 \%)$ & $<0.001$ \\
\hline Chemosis, n (\%) & $0(0.0 \%)$ & $11(10.9 \%)$ & $8(40.0 \%)$ & $2(25.0 \%)$ & 0.001 \\
\hline Discharge, n (\%) & $0(0.0 \%)$ & $1(1.0 \%)$ & $6(30.0 \%)$ & $4(50.0 \%)$ & $<0.001$ \\
\hline Trantas dot, n (\%) & $0(0.0 \%)$ & $0(0.0 \%)$ & $7(35.0 \%)$ & $2(25.0 \%)$ & $<0.001$ \\
\hline Ptosis, n (\%) & $0(0.0 \%)$ & $0(0.0 \%)$ & $1(5.0 \%)$ & $0(0.0 \%)$ & 0.016 \\
\hline Blepharitis, n (\%) & $0(0.0 \%)$ & $0(0.0 \%)$ & $0(0.0 \%)$ & $8(100 \%)$ & $<0.001$ \\
\hline Corneal ulcer, n (\%) & $0(0.0 \%)$ & $0(0.0 \%)$ & $5(25.0 \%)$ & $0(0.0 \%)$ & $<0.001$ \\
\hline
\end{tabular}

$p$-value $<0.05$ indicates statistical significance

Abbreviations: SAC, seasonal allergic conjunctivitis; PAC, perennial allergic conjunctivitis; VKC, vernal keratoconjunctivitis; AKC, atopic keratoconjunctivitis 


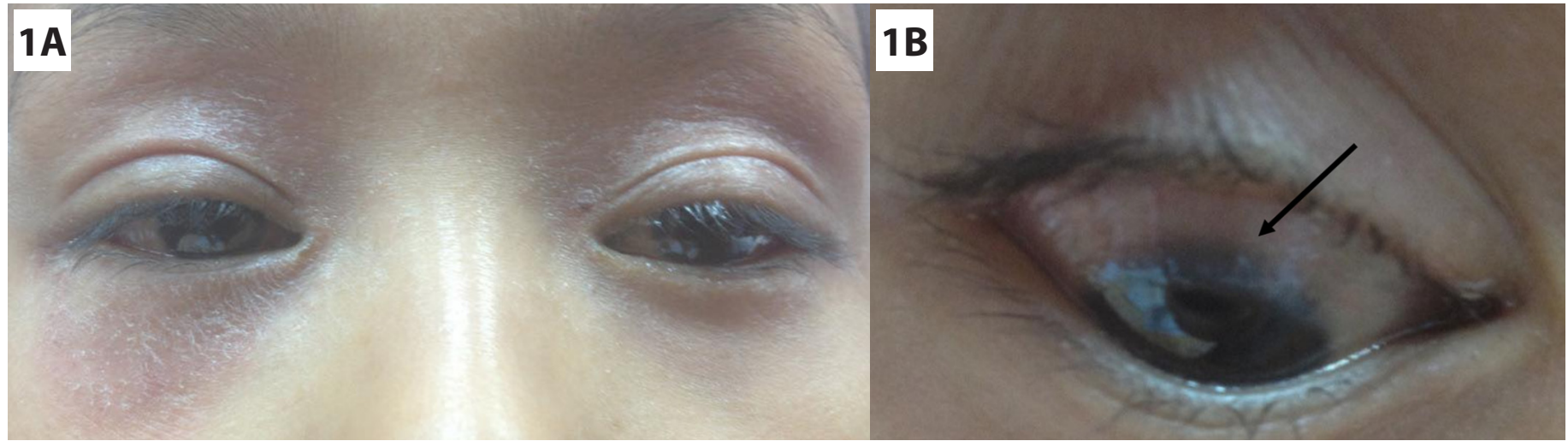

Figure 1. Patients with AKC (A) tearing and periorbital dermatitis (B) gelatinous infiltration at limbus

have palpebral typed (90\%) and only 2 patients have limbal typed (10\%). Most common signs of VKC were giant papillary, and Trantas dot. Interestingly, corneal ulcer (25\%) were found only in VKC patients. Furthermore, 1 patient in VKC group had unilateral ptosis, but it was reversible during 1 year of treatment. In contrast, blepharitis was found only in patients with AKC (Figure 1).

\section{Allergen sensitizations}

One hundred and forty-eight patients had aeroallergens sensitization. All AKC patients had sensitization to aeroallergen, whereas non-sensitization allergic conjunctivitis was found in SAC (22.9\%), PAC (7.9\%), and VKC (25\%). The most common sensitized allergens were house-dust mites (86.1\%), followed by cockroaches (47.9\%), pollens $(34.7 \%)$, animal dander (29.9\%), and molds (18.1\%). The distribution types of aeroallergen sensitization were not different between each type of ocular allergy (Table 3 ).

The number of allergen sensitization does not correlate with disease severity. Also, increasing of mean wheal size of both house dust mites and cockroaches are not asssociated with disease severity. Other atopic diseases are more common in mild type of $\mathrm{AC}(p=0.01)$

\section{Outcome of treatment}

The standard medications in all groups were topical olopatadine with artificial tear. Add-on medications were needed in severe form of AC. The history of topical corticosteroid use was $68.8 \%$ and $12.5 \%$ in $\mathrm{VKC}$ and $\mathrm{AKC}$ group, respectively. All of them can discontinue topical corticosteroid when topical tacrolimus was applied. Photophobia and lacrimation were resolved within the first few days. Also, Hyperemia and itching eyes were improved at the first few weeks. Significant reduction in number and size of giant papillary lesions after 1 month of tacrolimus treatment.

The outcome of treatment in VKC and $\mathrm{AKC}$ group are shown in Figure 2. In VKC group, subcutaneous immunotherapy was performed in 2 patients after using tacrolimus eye ointment. The median duration of treatment was 20.5 months, 45.5 months in complete remission and partial remission, respectively. The median time to relapse was 20.5 months. All 11 patients who continue tacrolimus ointment were well response and can continue tapering the dosage. Median duration to follow up of patients in this group was 8 months.

Table 3. Aeroallergen sensitization compared among different types of allergic conjunctivitis.

\begin{tabular}{|l|c|c|c|c|c|}
\multicolumn{1}{|c|}{ Aeroallergens } & $\begin{array}{c}\text { SAC } \\
(\mathbf{n}=\mathbf{3 5})\end{array}$ & $\begin{array}{c}\text { PAC } \\
(\mathbf{n}=\mathbf{1 0 1})\end{array}$ & $\begin{array}{c}\text { VKC } \\
(\mathbf{n}=\mathbf{2 0})\end{array}$ & $\begin{array}{c}\text { AKC } \\
(\mathbf{n}=\mathbf{8})\end{array}$ & $p$ \\
\hline Mites, n (\%) & $23(85.2 \%)$ & $82(88.2 \%)$ & $11(68.8 \%)$ & $8(100 \%)$ & 0.11 \\
Cockroaches, n (\%) & $12(44.4 \%)$ & $41(44.1 \%)$ & $10(62.5 \%)$ & $6(7.05 \%)$ & 0.23 \\
\hline Pollen, n (\%) & $11(40.7 \%)$ & $34(36.6 \%)$ & $3(18.8 \%)$ & $2(25.0 \%)$ & 0.47 \\
\hline Pets, n (\%) & $8(29.6 \%)$ & $29(31.2 \%)$ & $4(25.0 \%)$ & $2(25.0 \%)$ & 0.97 \\
Molds, n (\%) & $4(14.8 \%)$ & $20(21.5 \%)$ & $2(12.5 \%)$ & $0(0.0 \%)$ & 0.38 \\
\hline Negative, n (\%) & $8(22.9 \%)$ & $8(7.9 \%)$ & $5(25.0 \%)$ & $0(0.0 \%)$ & 0.27 \\
\hline
\end{tabular}

$p$-value $<0.05$ indicates statistical significance

Abbreviations: SAC, seasonal allergic conjunctivitis; PAC, perennial allergic conjunctivitis; VKC, vernal keratoconjunctivitis; AKC, atopic keratoconjunctivitis 

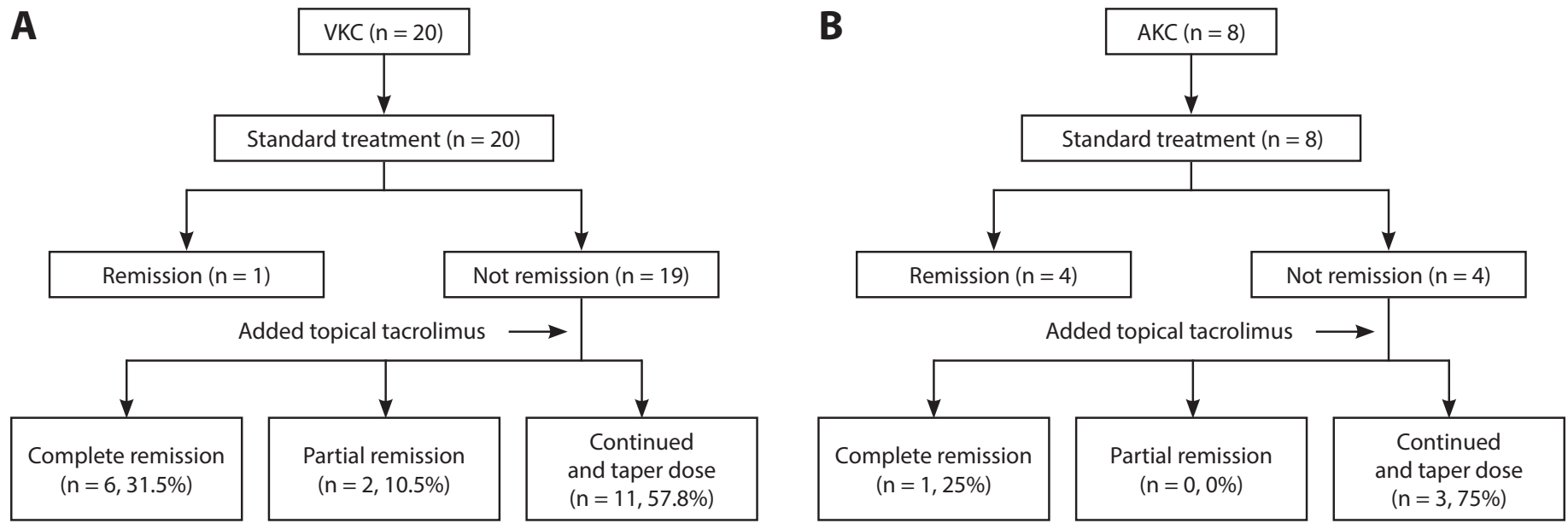

Figure 2. Prognosis of patients with severe conjunctivitis. (A) Vernal keratoconjunctivitis (VKC) (B) Atopic keratoconjunctivitis (AKC)

In AKC group $(\mathrm{n}=8)$, subcutaneous immunotherapy was performed in 3 patients who had continued tacrolimus treatment. The median duration of tacrolimus treatment was 11 months in complete remission group, and 18 months in non-remission group.

\section{Discussion}

Our study showed that children with ocular allergy mostly sensitized to house dust mites (86.1\%), followed by cockroaches (47.9\%), and pollens (34.7\%). In severe form of AC, all of them can discontinued topical corticosteroid when tacrolimus was applied. The overall remission was found $35 \%$ in VKC group and $63 \%$ in AKC group. The median duration of treatment was 20.5 months in VKC group and 11 months in AKC group.

Our study found that PAC is the most common type of ocular allergy in Thailand. This result can explain by the fact that Thailand is a tropical country and have less seasonal variation, compare to the studies from temperate climate. ${ }^{17}$ For this reason, it could be explain the type of allergen sensitization in our study as well. We found that most patients in all type of AC sensitized to house dust mite and cockroaches, not pollen. In contrast, the studies from Europe found that the majority of allergen sensitization was pollens. ${ }^{9,18}$ In addition, both humidity and temperature are proper to support mites and cockroaches well growing. Also, cockroaches is the second common allergen in our study that related with Tungtrongjitr and Sookrung study. ${ }^{19,20}$ Also a previous study in adult Thai population found that house dust mites and cockroaches are the major aeroallergen of allergic conjunctivitis. ${ }^{13}$ Therefore, the climate is the important factor for that affect type of allergen sensitization.

In SAC group, most patients suffered from the symptoms during rainy season (57.1\%), it might due to mite allergy because rainy season are proper to support mites growing. Also, the recent survey in Bangkok reported that the highest peak of pollen count was found in September. ${ }^{21}$ Whereas, the studies from western countries, ${ }^{4,6,22}$ the most common aeroallergen sensitization in SAC (spring, fall) is pollens.
According to demographic data, we found similar data like previous study ${ }^{10}$ such as male preponderance in all types, $60 \%$ of AC have family history of atopy and mean age of onset VKC (5.8 \pm 2.4 years $)$ seem to be earlier than other types. Interestingly, we found 8 patients (4.8\%) with AKC, mean age of onset $7.4 \pm 2.6$ years, which is contrast to previous studies ${ }^{22-24}$ suggested that AKC typically seen in the middle age (peak incidence 30-50 year) or late teenage. Therefore, AKC is not uncommon in children.

In this study, common manifestations in ocular allergy are itching, red eye and allergic shiner. Photophobia, giant papillary injection and Trantas dot were found in severe types. Blepharitis might be a specific sign of AKC, found only in AKC patients $(100 \% ; p<0.001)$, that is similar to a recent study from Fauquart. ${ }^{5}$ While Bielory study ${ }^{22}$ found $65.7 \%$ of patients. Also, Giant papillary and corneal ulcer were also found only in VKC patients. Interestingly, we found that one VKC patient had ptosis complication. The cause is unknown, Griffin $^{25}$ explained that it might be from the heavy giant papillae, an inflammation of the levator palpebrae superioris muscle that cause subsequent dis-insertion.

Our study found there is no correlation between number of allergen sensitizations and mean wheal diameters of specific allergen with the severity of disease. Because the mechanism of severe type of AC is not only IgE-mediated hypersensitivity, but also non IgE-mediated mechanism. We found that $25 \%$ of VKC patients had negative result for skin prick test.

The standard treatment in ocular allergy are allergen avoidance, artificial tear, topical antihistamine/mast cell stabilizer. Importantly, the proper allergic rhinitis treatment is crucial to control the eye symptoms. First, the congested nasal mucosa would obstruct the nasolacrimal duct drainage that causes allergen retention in the conjunctiva. ${ }^{26}$ Second, the histamine release in nasal mucosa stimulates nerves, then cause the ocular symptoms (naso-ocular reflex). ${ }^{27}$ 
Our study found that almost all of VKC patients needed add-on medication, whereas only half of AKC patients needed it. The history of topical corticosteroid use was $68.8 \%$ and $12.5 \%$ in VKC and AKC patients. Due to the risk of complication from topical corticosteroid use, our institute uses topical tacrolimus as an add-on medication for all patients with severe ocular allergy who did not respond to the standard treatment. We found that all of them can discontinue topical corticosteroid when topical tacrolimus was applied. So, we recommended the use of topical tacrolimus in patients with severe ocular allergy who did not respond to the standard treatment, especially those who were prescribed the topical corticosteroid. Although, the overall remission in our study was found only $35 \%$ in VKC group and 63\% in AKC group. This result was similar to previous studies. ${ }^{15,28}$ However, the median duration of our patients in continue using tacrolimus group were only 8 months in $\mathrm{VKC}$, and 18 months in AKC group, so the remission rate might underestimated.

Mahdy RA study ${ }^{29}$ showed that subcutaneous immunotherapy (SCIT) for mites or pollens are more effective than intermittent using topical steroid with combination eye drop in improving the clinical VKC symptoms after 24 months of treatment. We performed SCIT in 5 patients ( $\mathrm{VKC}=2$ patients, and $\mathrm{AKC}=3$ patients). All of them had to continued topical tacrolimus during the Median time to follow up were 14.5 and 22 months in VKC and AKC, respectively. Because the short follow-up time, so we cannot conclude the effect of SCIT in these patients.

The limitations of our study is a retrospective study and small population of severe form of $\mathrm{AC}$, so we cannot evaluate the long term outcome of the treatment with topical tacrolimus.

\section{Conclusion}

PAC is the most common type of AC in Thailand, and house dust mites is the most common aeroallergen in all 4 type of AC. The standard ocular treatments especially properly controlling allergic rhinitis symptoms were crucial for the AC treatment. However, the add-on medication is usually needed in severe forms of AC. Almost all of VKC patients needed add-on medication. Using topical calcineurin inhibitor as an add-on therapy, has a satisfactory result, and can decrease the use of topical corticosteroid. Atopic keratoconjunctivits could be found in childhood period. Furthermore they had better prognosis than VKC.

\section{Conflict of interest declaration}

The authors hereby declare no personal or professional conflicts of interest regarding any aspect of this study.

\section{Funding disclosure}

This study was supported by a Siriraj Grant for Research Development, Faculty of Medicine Siriraj Hospital, Mahidol University Bangkok, Thailand.

\section{References}

1. Singh K, Axelrod S, Bielory L. The epidemiology of ocular and nasal allergy in the United States, 1988-1994. J Allergy Clin Immunol. 2010; 126:778-83.

2. Asher MI, Montefort S, Bjorksten B, Lai CK, Strachan DP, Weiland SK, et al. Worldwide time trends in the prevalence of symptoms of asthma, allergic rhinoconjunctivitis, and eczema in childhood: ISAAC Phases One and Three repeat multicountry cross-sectional surveys. Lancet. 2006; 368:733-43.

3. Chinratanapisit S, Suratannon N, Pacharn P, Sritipsukho P, Vichyanond P. Prevalence and severity of asthma, rhinoconjunctivitis and eczema in children from the Bangkok area: The Global Asthma Network (GAN) Phase I. Asian Pac J Allergy Immunol allergy. 2018. Oct 15. doi: 10.12932/AP-120618-0336. [Epub ahead of print].

4. Leonardi A, Bogacka E, Fauquert JL, Kowalski ML, Groblewska A, Jedrzejczak-Czechowicz $M$, et al. Ocular allergy: recognizing and diagnosing hypersensitivity disorders of the ocular surface. Allergy. 2012;67:1327-37.

5. Fauquert JL. Diagnosing and managing allergic conjunctivitis in childhood: the allergist's perspective. Pediatr Allergy Immunol. 2019;30: 405-414.

6. Bielory L, Meltzer EO, Nichols KK, Melton R, Thomas RK, Bartlett JD. An algorithm for the management of allergic conjunctivitis. Allergy Asthma Proc. 2013;34:408-20.

7. Vichyanond P, Pacharn P, Pleyer U, Leonardi A. Vernal keratoconjunctivitis: a severe allergic eye disease with remodeling changes. Pediatr Allergy Immunol. 2014;25:314-22.

8. Pacharn P, Vichyanond P. Immunomodulators for conjunctivitis. Curr Opin Allergy Clin Immunol. 2013;13:550-7.

9. Almaliotis D, Michailopoulos P, Gioulekas D, Giouleka P, Papakosta D, Siempis T, et al. Allergic conjunctivitis and the most common allergens in Northern Greece. World Allergy Organ J. 2013;6:12.

10. Kosrirukvongs P, Visitsunthorn N, Vichyanond P, Bunnag C. Allergic conjunctivitis. Asian Pacific journal of allergy and immunology. 2001;19: 237-44.

11. Kumar S. Vernal keratoconjunctivitis: a major review. Acta Ophthalmol. 2009;87:133-47.

12. Vichyanond P, Kosrirukvongs P. Use of cyclosporine A and tacrolimus in treatment of vernal keratoconjunctivitis. Current allergy and asthma reports. 2013;13:308-14.

13. Labcharoenwongs $P$, Jirapongsananuruk $\mathrm{O}$, Visitsunthorn $\mathrm{N}$, Kosrirukvongs P, Saengin P, Vichyanond P. A double-masked comparison of $0.1 \%$ tacrolimus ointment and $2 \%$ cyclosporine eye drops in the treatment of vernal keratoconjunctivitis in children. Asian Pacific journal of allergy and immunology. 2012;30:177-84.

14. Leonardi A. Management of vernal keratoconjunctivitis. Ophthalmol Ther. 2013;2:73-88.

15. Pacharn $P$, Visitsunthorn $N$, Jirapongsananuruk $O$, Vichyanond $P$ Vernal keratoconjunctivitis (VKC) treated with $0.1 \%$ FK-506 ophthalmic ointment result of three years follow-up. J Allergy Clin Immunol 2007;119:S153.

16. Kosrirukvongs P, Vichyanond P, Wongsawad W. Vernal keratoconjunctivitis in Thailand. Asian Pacific journal of allergy and immunology. 2003;21:25-30.

17. Leonardi A, Castegnaro A, Valerio AL, Lazzarini D. Epidemiology of allergic conjunctivitis: clinical appearance and treatment patterns in a population-based study. Curr Opin Allergy Clin Immunol. 2015;15: 482-8.

18. Leonardi A, Piliego F, Castegnaro A, Lazzarini D, La Gloria Valerio A, Mattana P, et al. Allergic conjunctivitis: a cross-sectional study. Clinical and experimental allergy : journal of the British Society for Allergy and Clinical Immunology. 2015;45:1118-25.

19. Tungtrongchitr A, Sookrung N, Munkong N, Mahakittikun V, Chinabut P, Chaicumpa W, et al. The levels of cockroach allergen in relation to cockroach species and allergic diseases in Thai patients. Asian Pacific journal of allergy and immunology / launched by the Allergy and Immunology Society of Thailand. 2004;22:115-21.

20. Sookrung N, Chaicumpa W. A revisit to cockroach allergens. Asian Pacific journal of allergy and immunology / launched by the Allergy and Immunology Society of Thailand. 2010;28:95-106. 
21. Songnuan W, Bunnag C, Soontrapa K, Pacharn P, Wangthan U, Siriwattanakul U, et al. Airborne pollen survey in Bangkok, Thailand: A 35-year update. Asian Pacific journal of allergy and immunology. 2015;33:253-62.

22. Bielory L. Allergic and immunologic disorders of the eye. Part II: ocular allergy. J Allergy Clin Immunol. 2000;106:1019-32.

23. Ono SJ, Abelson MB. Allergic conjunctivitis: update on pathophysiology and prospects for future treatment. J Allergy Clin Immunol. 2005;115:118-22.

24. Barney NP CE, Stahl JL. Allergic and Immunologic Diseases of the Eye. In: Adkinson NF, Bochner BS, Burks AW, Busse WW, Holgate ST, Lemanske RF, O'Hehir RE,editors. Middleton's Allergy principle and practice. $8^{\text {th }}$ ed: Elsevier Saunders; 2013. p. 618-37.
25. Griffin RY, Sarici A, Unal M. Acquired ptosis secondary to vernal conjunctivitis in young adults. Ophthalmic Plast Reconstr Surg. 2006;22: $438-40$.

26. Bernstein DI, Levy AL, Hampel FC, Baidoo CA, Cook CK, Philpot $\mathrm{EE}$, et al. Treatment with intranasal fluticasone propionate significantly improves ocular symptoms in patients with seasonal allergic rhinitis. Clin Exp Allergy. 2004;34:952-7.

27. Bielory L. Allergic conjunctivitis and the impact of allergic rhinitis. Curr Allergy Asthma Rep. 2010;10:122-34.

28. Bonini S, Coassin M, Aronni S, Lambiase A. Vernal keratoconjunctivitis. Eye (Lond). 2004;18:345-51.

29. Mahdy RA, Nada WM, Marei AA. Subcutaneous allergen-specific immunotherapy versus topical treatment in vernal keratoconjunctivitis. Cornea. 2012;31:525-8. 